\title{
Perception of bitterness, sweetness and liking of different genotypes of lettuce
}

Article

Accepted Version

Chadwick, M., Gawthrop, F., Michelmore, R. W., Wagstaff, C. and Methven, L. (2016) Perception of bitterness, sweetness and liking of different genotypes of lettuce. Food Chemistry, 197. pp. 66-74. ISSN 0308-8146 doi:

https://doi.org/10.1016/j.foodchem.2015.10.105 Available at https://centaur.reading.ac.uk/44991/

It is advisable to refer to the publisher's version if you intend to cite from the work. See Guidance on citing.

To link to this article DOI: http://dx.doi.org/10.1016/j.foodchem.2015.10.105

Publisher: Elsevier

All outputs in CentAUR are protected by Intellectual Property Rights law, including copyright law. Copyright and IPR is retained by the creators or other copyright holders. Terms and conditions for use of this material are defined in the End User Agreement.

\section{www.reading.ac.uk/centaur}

\section{CentAUR}

Central Archive at the University of Reading 
Reading's research outputs online 


\section{Perception of bitterness, sweetness and liking of different}

genotypes of lettuce.

\section{Chadwick $\mathbf{M}^{1,3}$, Gawthrop F3, Michelmore RW ${ }^{4}$, Wagstaff $\mathrm{C}^{1,2^{*}}$}

\section{and Methven $\mathbf{L}^{1}$.}

${ }^{1}$ Department of Food and Nutritional Sciences, University of Reading, PO Box 226,

Whiteknights, Reading, Berkshire, RG6 6AP, UK.

${ }^{2}$ Centre for Food Security, University of Reading, Whiteknights, Reading, Berkshire, RG6 $6 A H, U K$.

${ }^{3}$ Tozer Seeds, Pyports, Downside Bridge Road, Cobham, Surrey, KT11 3EH, UK.

${ }^{4}$ The Genome Center, 1 Shields Avenue, University of California, Davis, CA 95616, USA.

*Author for correspondence. Email: c.wagstaff@reading.ac.uk

Keywords: lettuce, sesquiterpenoid lactone, bitterness, sweetness, sugar, bitter suppression 


\section{Abstract}

2 Lettuce is an important leafy vegetable, consumed across the world, containing bitter

3 sesquiterpenoid lactone (SL) compounds that may negatively affect consumer acceptance

4 and consumption. We assessed liking of samples with differing absolute abundance and

5 different ratios of bitter:sweet compounds by analysing recombinant inbred lines (RILs)

6 from an interspecific lettuce mapping population derived from a cross between a wild ( $L$.

7 serriola acc. UC96US23) and domesticated lettuce, (L. sativa, CV. Salinas). We found that the

8 ratio of bitter:sweet compounds was a key determinant of bitterness perception and liking.

9 We were able to demonstrate that SLs such as 8-deoxylactucin-15-sulphate contribute most

10 strongly to bitterness perception, whilst 15-p-hydroxylphenylacetyllactucin-8-sulphate does

11 not contribute to bitter taste. Glucose was the sugar most highly correlated with sweetness

12 perception. There is a genetic basis to the biochemical composition of lettuce. This

13 information will be useful in lettuce breeding programmes in order to produce leaves with

14 more favourable taste profiles. 


\section{Introduction}

Sesquiterpene lactones are anti-feedants and phytoalexins produced by lettuce (Lactuca sativa L.). Selective breeding against the bitter taste imparted by them has reduced presence of these compounds in domesticated lettuce cultivars dramatically (Wink, 1988). Many modern varieties do still contain perceivable quantities of sesquiterpene lactones and this is particularly relevant with a move away from iceberg-type head-lettuce to bagged lettuce which contain fewer high yielding, sweet cultivars and more red-leaved varieties, which typically contain much higher concentrations of the bitter compounds (Price et al., 1990). The perceived bitterness is enough to reduce palatability and consumption in a westernised diet, where fruit and vegetables are already under-consumed (Casagrande et al., 2007; Rogers and Pryer, 2012). It is widely believed that this bitterness can be counteracted by sweetness (Bartoshuk, 1975; Keast and Breslin, 2003); an improvement in flavour is therefore likely to be a consequence of manipulating both factors. Although sensory perception of individual sugars (Pangborn, 1963) and SLs (Price et al., 1990; Seo et al., 2009; Sessa et al., 2000) has been previously assessed and sensory perception is well established in the case of sweet compounds, assessment of SL bitterness is sometimes contradictory and has not been considered with regards to tastant mixture suppression. Here we assess the interaction between sweet and bitter components within the natural food matrix of lettuce and additionally compare perception data to consumer liking.

Lettuce is a suitable crop in which to pursue flavour improvement as it is widely eaten across Europe and North America. Lettuce also contains a range of beneficial secondary plant metabolites including, phenolics, ascorbate, $\alpha$-tocopherol, lignans, as well as SLs (García-Macías et al., 2007; Oh et al., 2009); consequently improving the flavour should 
increase consumer intake. Phytochemicals present in lettuce have been suggested as having a range of biological functions, from analgesic, anti-inflammatory, anti-tumour, and gastroprotective effect of the sesquiterpenoids (Giordano et al., 1990; Guzman et al., 2005; Sayyah et al., 2004), to cognitive effect of phenylpropanoid flavonoids (García-Macías et al., 2007; Spencer et al., 2009). Additionally lettuce, particularly the romaine type, is a source of iron and potassium and a good source of dietary fibre, folate and manganese, vitamins A, B1, B6, C, K, and omega-3 fatty acids (Belitz et al., 2009). Bitterness in lettuce is not thought

47 to be linked to the beneficial biological effects of the same molecules, owing to distinct functional groups in the compounds (Chadwick et al., 2013; Behrens et al., 2009; Brockhoff et al., 2007) and so it is feasible to balance the reduction of those most bitter SLs while maintaining or increasing those with greatest biological function.

Sweet and bitter tastes are sensed through the binding of the tastants to G-protein coupled receptors located within papillae on the tongue. Sugars bind to type 1 receptors (T1R) (Meyers \& Brewer, 2008) and bitter molecules to type 2 receptors (T2R) (Meyerhof, Batram, Kuhn, Brockhoff, Chudoba, Bufe, et al., 2010). Whereas there are just two T1R receptors involved in sweet perception (T1R2/T1R3) there are 25 T2Rs responsible for binding a broad range of bitter molecules. Whereas some T2Rs are generalists and bind to a wide range of structurally diverse molecules, others are specialists binding to a narrow range of compounds (Meyerhoff et al., 2010). SLs have been found to activate the T2R46, a generalist receptor (Brockhoff et al., 2007). Within the population it is common to categorise individual as "bitter sensitive" or "bitter blind", where $25 \%$ of the population are "bitter blind" , however this categorisation is due to polymorphisms of the Tas2R38 gene (Menella et al., 2010). The receptor T2R38 is a specialist receptor binding to thiouracil groups (as 
found in Brassica vegetables) and not to SLs. We therefore propose that "bitter blindness" resulting from Tas2R38 will not effect consumers perception of bitterness in lettuce.

We hypothesise that consumers are able to accurately detect sweetness and bitterness in lettuce as imparted by the compounds of interest. We also propose that taste interaction between sweetness and bitterness as well as the absolute concentrations of the compounds will have a significant effect on taste perception and liking. Additionally, it is broadly believed that consumers prefer foods which they perceive as sweet. To most consumers a major factor in purchasing habits is liking for taste (Enneking et al., 2007) and so ultimately this will be the chief factor in delivering a positive change in consumer habits.

\section{Materials and Methods}

\section{Plant Material and Growth Conditions.}

Fg recombinant inbred lines (RILs) were supplied by the Michelmore lab (Genome Center, UC Davis, USA) and 102 RILs plus their parents, L. sativa cv. Salinas and the wild L. serriola UC96US23, were propagated by A.L. Tozer. For these studies, plants were grown under glasshouse conditions at The University of Reading and watered once or twice daily in accordance with the weather. The glasshouse temperature ranged from 17 to $30^{\circ} \mathrm{C}$. Seedlings were transferred from seed trays to $3 \frac{1}{2}$ " pots with Osmocote after 3 weeks, and were given Sangral 1:1:1 liquid fertiliser weekly. Plants were harvested after 49 days, at a mature, commercially viable, stage and prior to floral transition.

The 102 RILs were analysed by HPLC-MS (see section below) to assess SL abundance and sugar assays to assess the concentrations of sucrose, fructose and glucose (see section below) in order to determine which lines would be most informative. Eight RILs were 
selected based on whether that line had high or low concentrations of sugar and SLs. The sample size was kept small to avoid fatigue in the consumer panel.

\section{Consumer Analysis and Sample Preparation}

88 Lettuce samples were harvested daily on the morning of the tests and were used within an hour of preparation, being kept refrigerated and moist until they were needed in order to reduce respiration and sample wilting. Leaf samples were cut into strips $5 \mathrm{~cm}$ by $1 \mathrm{~cm}$, avoiding the midrib as this can contain more variable levels of SLs (Sessa et al., 2000).

Samples were labelled with arbitrary three digit codes in petri-dishes and three strips were provided per consumer. All consumer work took place in sensory booths at the University of Reading, with neutral odour, artificial daylight and controlled temperature. Forty three consumers took part in the study, consisting of eight men and 35 women. Ages ranged from 17 to 68 with 6 over the age of 40 (mean $=29.8$ years, median $=25$ years). This skew in participant age was due to primary recruitment taking place on the university campus. Participants were recruited after ethical approval of the study (University of Reading Research Ethics Committee, study number 08/13) via email notification and poster advertisement and volunteers were screened by questionnaire for any dietary restrictions,

101 allergies or health conditions that may have affected their ability to participate the consumer study.

Consumer response was recorded using Compusense 5 software (Compusense Ontario,

104 Canada). The study was divided into three sections. First, participants were asked to familiarise themselves with a labelled magnitude scale, rating their most bitter, sweet, salty and sour experiences on the scale. This was used to normalise their scores against other participants, to allow for high and low scale users. The main study involved rating lettuce 
samples presented to them one at a time in a balanced design for liking on a 9 point hedonic category scale (anchored from dislike extremely to like extremely), then for perception of sweetness and bitterness using labelled magnitude scales (where semantic descriptors from weak to strongest imaginable are positioned on a logarithmic scale, and scored 0 to 1.97).

112 Participants were asked to taste each sample three times, once for liking, then sweetness and again for bitterness. Finally perception of aftertaste intensity was rated on a 5 point

114 category scale (anchored from no after taste to very strong) after a 10 second wait period.

115 Participants were also asked to give any additional comments on the samples. Once the 116 assessment of one lettuce line was completed, participants were given the next sample

117 after a 30 second rest period. Participants were given water and plain water crackers

118 (Carr's, United Biscuits, UK) to cleanse their palate during this rest period. See

119 supplementary data for a transcript of the questions exactly as posed. After the test

120 participants were given an exit questionnaire asking for further information on age, gender,

121 frequency with which they consume lettuce, and also the regularity of their consumption of

122 bitter foods in their diet, based on a list of 12 common bitter foods (white cabbage, green

123 cabbage, red cabbage, cauliflower, kale, brussels sprouts, watercress, rocket, radish, coffee,

124 tonic water, and broccoli). Finally they were phenotypically tested for bitter blindness using 125 PTC (Phenylthiocarbamide) strips. Bitter blindness occurs in around $25 \%$ of people as the 126 result of an inactive hTAS2R38 receptor and, while it is not directly responsible for detection 127 of SLs, it is a widely accepted indicator of bitter taste acuity.

\section{Chemical analysis}

129 Sesquiterpene lactones and some polyphenols in the main population of 102 RILs were analysed by HPLC and identities confirmed by HPLC-MS based on details published in Sessa et al. (2000), mass data for each compound was as follows; Lactucin m/z 277; Lactucopicrin 
$\mathrm{m} / \mathrm{z} 411 ; 8$-deoxylactucin $\mathrm{m} / \mathrm{z}$ 332; 15-p-hydroxyphenylacetylactucin-8-oxalate $\mathrm{m} / \mathrm{z}$ 490;

133 Lactucin oxalate $\mathrm{m} / \mathrm{z}$ 348; Lactucopicrin oxalate $\mathrm{m} / \mathrm{z} 482$. Full spectra are presented in

134 Supplementary Figure 1. Plant samples from each individual genotype were replicated in 135 quadruplicate and analysed individually for determination of SLs. These were extracted as

136 follows: $0.5 \mathrm{~g}$ of frozen homogenised leafy plant material was added to $2 \mathrm{ml}$ of $70 \% \mathrm{MeOH}$,

137 shaken for 10 minutes, centrifuged $\left(13000 \times \mathrm{g}, 4^{\circ} \mathrm{C}, 5 \mathrm{~min}\right)$ and filtered through a $0.45 \mu \mathrm{m}$

138 filter attached to a syringe; the supernatant was run in an Agilent 1100 HPLC system (Agilent

139 Technologies, Wokingham, UK) coupled to a Bruker Microtof high resolution quadrupole-

140 time of flight mass spectrometer (QToFMS) (Bruker Daltonics Ltd, Coventry, UK). Samples

141 were separated on an ACE $C_{18} 15 \mathrm{~cm} \times 2.1 \mathrm{~mm}, 5 \mu \mathrm{m}, 100 \AA ̊$ HPLC column (Advanced

142 Chromatography Technologies, UK). Running conditions were as according to Table S1 with

143 a flow rate of $0.5 \mathrm{ml} / \mathrm{min} ; 50 \mu \mathrm{l}$ injection and UV response measured at $264 \mathrm{~nm}, 280 \mathrm{~nm}$,

$144320 \mathrm{~nm}$, and 365nm, 520nm.

145 Sugars were assessed by high throughput plate assays using a modified version of Wingler et al. (2006). Lettuce samples were first weighed and solutes extracted by heating to $80^{\circ} \mathrm{C}$ in

$14780 \%$ ethanol; the supernatant was dried under vacuum (Savant Speed Vac, Thermo

148 Scientific, MA, USA). Sugars were then resuspended in $100 \mu$ l of sterile deionised water.

149 Sugars were assessed by hexokinase (Roche; 1500units/ml diluted 1:30 in HEPES buffer)

150 directed phosphorylation of glucose, leading to reduction of NAD+ to NADH whereupon a

151 change in absorbance at 340nm proportional to sugar content can be measured. Sucrose

152 was converted to glucose by hydrolysis of sucrose by invertase (Sigma; 355 units/ml diluted

$1531: 150$ in HEPES buffer) and fructose-6-phosphate converted to glucose-6-phosphate by

154 phosphoglucose isomerase (Roche; $2 \mathrm{mg} / \mathrm{ml}$ diluted 1:10 in HEPES buffer). 


\section{Statistical Analysis}

156 In order to determine whether there were significant differences in consumer perception

157 and liking between the RILs, response data were normalised and assessed for variance by

158 Kruskal-Wallis with Dunn's procedure. Correlation statistics assessed by Spearman's rank

159 were completed using Prism 6 (GraphPad Software, Inc., La Jolla, USA). Significant

160 differences were determined at $95 \%$ confidence intervals $(P<0.05)$. An internal preference

161 map was attained by carrying out a principle component analysis of the individual liking data

162 and fitting the mean ratings for bitter and sweet perception, as well as the mean liking

163 ratings, onto the plot as supplementary variables using XLStat (AddinSoft, version 2012.1.01,

164 Paris, France).

\section{Results}

\section{Sample Selection}

167 Lines within the mapping population were selected for extreme values in concentrations of sugars, total SLs, and for specific SLs according to previously reported bitterness ratios. This was done to maximise qualitative data from a small number of samples, hence while others were selected for overall profile, RILs 41 and 122 were selected on account of having

171 particularly high concentrations of lactucin-15-oxalate, which was reasoned to be the most

172 bitter individual SL based on correlation data in previous research by Price et al. (1990).

173 Absolute concentration of each assessed compound is given in Table S2, along with the

174 rationale for the RIL's selection.

\section{Demographic factors}

176 Regularity of lettuce consumption was ascertained by individual recall. There was no

177 significant link to perception of bitterness, nor to liking of certain samples. Of the 
participants only a single participant reported never eating lettuce, while 19 responded with

179 'more than once per week' which was the highest category on our scale. There was no

180 trend for participants who regularly consumed lettuce to prefer bitter or sweeter

181 genotypes, nor did this show any influence on bitter perception; however, the study size

182 was not large enough to conclude whether preference for bitter or sweet genotypes

183 influences frequency of lettuce purchase or consumption.

184 Volunteers reported the regularity with which they consumed other bitter foods. Frequency

185 of consumption of foods with known bitter components, such as a range of Brassicaceous

186 plants, coffee, and tonic water, were assessed and related to liking and perception scores,

187 with the conclusion that this does not affect preference for lettuce genotypes, nor does it

188 alter perception of bitterness or sweetness, within the population assessed. These findings

189 were anticipated, as there is little relationship between the SL structure and those of

190 brassica glucosinolates, or alkaloids such as caffeine present in coffee, or quinine used to

191 flavour tonic water; such compounds typically have a range of different structures and bind

192 to structurally different receptors. Age and gender data were also recorded, with no

193 significance found across age groups or gender. Finally, participants were tested for bitter

194 blindness using PTC strips. Eleven volunteers were found to be bitter blind, while the

195 remaining 32 were tasters, as predicted for a Mendelian segregation of a phenotype

196 controlled by a single gene. There was no significant difference between liking or

197 perception scores of either bitter tasting or non-tasting consumer groups, indicating that,

198 unlike hTAS2R46, the hTAS2R38 receptor has no role in detecting the bitter taste derived 199 from SL compounds. 
201 We found that there was significant variation in reported bitterness, sweetness, aftertaste

202 and in consumer liking between different lines (Figure 1). This showed that consumers were able to detect the differences between the samples in terms of the major sensory parameters related to sesquiterpene lactones and to sugars; bitterness, aftertaste, sweetness, and these attributes influenced preference. The perceived bitterness and sweetness correlated to absolute phytochemical levels with high statistical significance in most cases.

In terms of sweetness perception (Figure 2) consumers ranked RILs 41 and 123 as the least sweet, and these RILs indeed had relatively low sugar contents, however RIL 61 also had a very low total sugar content and was rated relatively high for sweetness. RIL 61 has the lowest levels of fructose, the sugar with the highest relative sweetness of the sugars present in this lettuce population. This was expected therefore the be perceived as least sweet, however it also contained the least total SL content of all the tested samples, showing that interaction of the bitter SLs suppressed the sweetness of the other lines (Figure 3A). The sweetness of RIL 61 can largely be attributed to its glucose content, which was considerably higher than either fructose or sucrose. Glucose levels had the greatest correlation with perceived sweetness $(r=0.2266 P<0.0001)$ across all lines, possibly because it was the most abundant sugar in the lines perceived as being the most sweet (RILs 61 and 19). RILs 41 and 123 were significantly the least liked and perceived as the least sweet (at $p<0.05$ ); these samples had significantly less sugar than the other selected lines in terms of total sugar and

221 for each of the individual sugars tested for. Correction for the relative sweetness of each

222 sugar present (glucose; 0.74 , sucrose; 1, fructose; 1.73; Koehler and Kays, 1991) was used to 223 determine an expected total sweetness level (Figure 2E). This shows a positive relationship 
between perceived sweetness and relative sucrose equivalent concentration $(r=0.961, P=-$

0.002) despite other confounding effects, such as influence of bitterness. RILs 61 and 122

maintain higher perceived sweetness compared to predicted sweetness scores, due to their

227 relatively low concentration of total SLs, at a factor of 4-24 fold less than RIL 123 which had

228 the highest SL content. RIL 94, which was selected for high concentration of SLs in combination with high sugar content, was marginally less sweet than might be anticipated

230 from sugar content alone, owing to sweetness suppression by the bitter compounds.

231 Consumers perceived RIL 123 and 41 (selected for high total SL and high lactucin-15-oxalate respectively) as the most bitter, significantly different from all others $(P=<0.0001$; Figure 3$)$.

233 Of our detected SLs, only 15-p-hydroxylphenylacetyllactucin-8-sulphate (Figure 3G) showed no correlation with bitterness, while 8-deoxylactucin-15-sulphate (Figure 3B) showed the most divergence between lines and had the strongest positive relationship when content was correlated with bitter perception, suggesting that this is the compound which most strongly drives the bitter taste in our lettuce population. The sample perceived to be least bitter compared to the others was RIL $61(\mathrm{P}<0.0001)$, consistent with it having the lowest concentrations of most SLs, including 8-deoxylactucin-15-sulphate, and the least total SL content. The low SL content also means that there would be less suppression of sweet taste, hence the higher than anticipated sweetness perception for this line even though it

242 had low sugars (which can mask bitterness) (Figure 2E).

\section{Consumer Liking}

244 Spearman correlation was conducted to relate liking to perception of each of the 3 sensory attributes. Sweetness was seen as the main positive influence on liking $(r=0.40, P<0.0001$;

246 Figure 4A), whereas perceived bitterness gave a strong negative correlation ( $r-0.56$, 
$\mathrm{P}<0.0001 ;$ Figure 4B). Consumers' perceptions accurately matched the chemical analysis,

once both bitter and sweet compounds were considered together, and have highlighted the differences between compounds in terms of their contribution to overall taste perception. For this reason, RIL 61 was the most liked sample, despite the fact that it does not have the highest sugar content or the lowest content of every SL. Aftertaste perception was negatively correlated to liking $(r=-0.31, P<0.0001)$, and SL content $(r=-0.27, P<0.0001$; Figure 4C) and positively correlated with bitterness perception ( $r=0.61, P<0.0001)$. Aftertaste was correlated to all SLs with the exception of 15-p-hydroxylphenylacetyllactucin-8-sulphate ( $r=$ $0.07687 \mathrm{P}=0.1835)$, which was the compound which did not appear to have any association with bitter taste, but correlated best with 8-deoxylactucin-15-sulphate $(r=0.2687 \mathrm{P}=<$ 0.0001) which was the most bitter compound. RIL 61 was perceived as imparting significantly less aftertaste than the other samples, while RILs 123 and 41 grouped as imparting the most aftertaste, implying that the most bitter compounds are the principle contributors to aftertaste. Consequently, we can assume that modifying concentrations of these compounds in novel cultivars will have a perceivable positive effect on consumer

262 liking.

263 Our consumers reported that they most liked RILs 19, 61 and 89, and disliked the RILs 41 and 123. RILs 19 and 89 had the highest total sugar content, while 61 was selected on account of having low total SL content, explaining preference for these samples over others. RILs 41 and 123 were perceived as the most bitter as well as being the least liked; 41 was

267 selected for high lactucin-15-oxalate, while 123 was selected for high total SL content. A preference map was derived using principle component analysis to relate the consumer perception of the taste attributes to the individual consumer liking ratings (Figure 5), where 

the positioning of the samples on the map is derived from the individual liking data. This

271 showed an overall preference for sweeter lines and dislike for bitter lines with the first

272 principal component accounting for $28.7 \%$ of the variance in liking. The secondary principal

273 component, accounting for $19.7 \%$ of the variance, was not related to any of the assessed

274 parameters, and may not relate to taste, but another sensory parameter such as colour or

275 texture. Dimensions 3 and 4 accounted for $29.2 \%$ of the variance (plot not shown),

276 dimension 3 separated RILs 89 and 121 and dimension 4 separated RILs 19 and 61, where in

277 both cases these RILs were positioned together on PC1 and 2. This shows that no all

278 consumers gave them equal liking scores although, in both cases, their mean liking ratings

279 were not significantly different (see Fig 1). Interestingly in Figure 4, RIL 94, which contained

280 both high SLs and high sugar, fell in the centre of the PCA and in the middle grouping for

281 preference, supporting the concept that high sugar concentrations do help to counteract

282 high SL content.

\section{Tastant Mixture Suppression}

284 Mixing suppression is thought to influence taste perception in food samples. Liking, bitterness perception, and sweetness perception were plotted against sugar to sesquiterpene ratios (Figure 6). There was a negative correlation between sugar:SL ratio and perceived bitterness $(r=-0.280, P<0.0001)$ and a weaker correlation between sugar:SL ratio and perceived sweetness $(r=0.171, P=0.0015)$, although liking was not significant with sugar:SL ratio $(r=0.042, P=0.2338)$. Taking mixing suppression into account moved outliers such as RIL 61 back toward their anticipated ranking of sweetness perception relative to the other RILs, confirming that mixing interaction between the taste factors was driving overall perception of taste. 


\section{Discussion}

295 Sesquiterpene lactones in a natural food matrix can impart a bitter taste to consumers, with our consumer panel reliably scoring samples correctly in terms of bitterness with regards to the quantities of their determining compounds as derived by biochemical measurement using HPLC. While it is known that there is great variety in the detection threshold between individual SLs, there is some disagreement as to which SL is the most influential on taste

300 (Sessa et al., 2000; Van Beek et al., 1990). We found that our consumers' bitterness detection positively correlated to 5 of the 6 SLs present in our samples as determined previously by HPLC and confirmed by HPLC-MS/MS. The only SL from our population not found to be correlated to bitterness was 15-p-hydroxylphenylacetyllactucin-8-sulphate, which makes it a strong candidate for counterbalancing any reduction in other SLs as it is unlikely to impart a perceptible change in bitterness. Maintaining concentration of less bitter SLs will potentially keep the analgesic and anti-inflammatory function of lettuce for consumers (Bork et al., 1996). This strategy may also retain the ability of the plant to survive field stress through the anti-feedant (Bennett, 1994; Cowan, 1999) and antimicrobial activities of the SL (Koul, 2008; Wedge et al., 2000), depending on whether herbivores and microbes are using the same mechanism as humans to structurally detect and respond to

311 individual SL compounds. Consumers were able to accurately rate bitter taste based on the content of SLs and primarily 8-deoxylactucin-15-sulphate, which has been previously rated

313 as one of the most bitter of the SLs present in lettuce (Peters and van Amerongen, 1998;

314 Price et al., 1990). The perceived sweetness scores correlated with total sugar content, but

315 the primary factor appears to be glucose content, which accounts for the majority of lettuce 
sugar content, despite fructose being the sweetest of the sugars present. It has been

317 reported that fructose is detected as $173 \%$ as sweet as sucrose on a pro rata basis and

318 glucose is considered the least strong tasting with a relative sweetness $74 \%$ that of sucrose

319 (Pangborn, 1963). The correlation between sugar concentrations and perceived sweetness

320 was less strong than that of SL concentrations and perceived bitterness. It is important to

321 consider the availability of compounds to taste receptors as a result of the natural food

322 matrix, which is not currently known for lettuce, and may vary with physiological

323 composition of the samples. Other interactions such as the effect of acidity on

324 complementing sweetness may also play a part as it does in tomato fruit (Baldwin et al.,

325 2008); however, acidity was not directly assessed for the present study. Additionally, cross-

326 modal interactions of small volatile molecules such as geranial and apocarotenoids are

327 thought to impact on the perceived sweetness of fruit (Green et al., 2010; McMath et al.,

328 1991; Tieman et al., 2012) and are also likely to affect the taste of lettuce.

329 It is important to consider the relative quantities, as well as simple detection thresholds, in

330 determining the net flavour profile of a food. RIL 61, which contained the second lowest

331 total sugars (only $40.6 \%$ that of RIL 19 , the highest total sugars) and the lowest total SLS

332 (6.1\% that of RIL 94) was consistently rated as one of the sweetest varieties and was rated

333 the most sweet overall. The most likely explanation is that there is a lower suppression

334 effect of bitter SLs on the sweetness of the sugars, leading to an increased perception of the

335 sugars present. In contrast, RIL 122, which had high concentrations of many SLs, was

336 perceived as less bitter than may be predicted on account of low content of 8-

337 deoxylactucin-15-sulphate which the SL most strongly correlated to bitterness. Price (1990)

338 and van Beek (1990) did not assess the conjugated form of this compounds but this is in 
dissent with their findings, which implicate lactucopirin as the most bitter SL backbone, but

340 is in keeping with their conclusion that conjugated forms of SLs are more bitter than those

341 which are not.

342 Taste perception is known to deteriorate with age, especially with regards to bitterness

343 perception due to its natural association with harmful toxins which are a presumed as a

344 greater hazard to children (Mennella et al., 2010), but we lacked the sample size and range

345 of ages to look into this further. We were also unable to determine gender differences

346 though there is some indication that women are more likely to be 'supertasters' and

347 therefore have increased taste and flavour perception on a population level (Bartoshuk et

348 al., 1994; Doty et al., 1985). We also looked at how regular consumption of bitter foods

349 affects bitterness perception, with regards to sensitisation due to frequent exposure to

350 bitter flavours, or a tolerance factor for the same reasons. We found that there was no

351 significant change in either direction; however, there was a trend toward people who

352 infrequently ate lettuce to prefer sweeter lettuce, possibly accounting for their lack of

353 consumption. This subgroup remains an important target group for marketing novel,

354 sweeter varieties. Some breeding to this end has already taken place resulting in the

355 commercially available Little Gem and O' So Sweet varieties which are small and sweet

356 romaine type lettuces. Bitter blindness to PTC had no effect on perception of bitter SLs. It is

357 known that the receptor involved in detection of sesquiterpene lactones is separate to that

358 which detects glucosinolates and which can cause 'bitter blindness' in $25 \%$ of people in

359 response to glucosinolate-derived compounds. The receptor known as hTAS2R46 has been

360 reported to be responsible for detection of SL compounds and other bitter substances, such

361 as clerodane and labdane diterpenoids, strychnine, and denatonium (Brockhoff et al., 2007). 
Kim et al. (2005) found that there are inactive polymorphisms of the HTAS2R46 receptor,

363 which would result in bitterness insensitivity in around $24 \%$ of the general population;

364 however, inability to detect sesquiterpene lactones has not been reported.

365 This study supports our hypothesis that consumers are capable of detecting the sweet and

366 the bitter compounds in lettuce, as well as our hypothesis that most consumers have a

367 preference for sweeter and less bitter genotypes. Our data suggest that the bitter and

368 sweet components act to counterbalance each other and that ratios of key compounds are

369 more important drivers of taste perception than concentrations of individual metabolites. It

370 is not entirely understood what the functional groups involved in SL bitterness are, but the

371 dienone system has been implicated (Ivie et al., 1975), in addition to steric interference

372 from other large modifications to the primary SL backbones. However, it is commonly

373 accepted that the while biological function is primarily attributed to the $\alpha M \gamma L$ group,

374 bitterness is not (Brockhoff et al., 2010). The fact that the SLs show varying degrees of

375 influence on bitter perception, with one SL showing no significant correlation, is therefore a

376 promising result. 8-deoxylactucin-15-sulphate showed the strongest correlation to

377 perceived bitterness in this study where the oxalates of lactucin and lactucopicrin were also

378 strongly correlated with bitterness, consistent with previous reports (Peters and van

379 Amerongen, 1998; Van Beek et al., 1990).

380 Lettuce breeding programmes should therefore target an increase in sugar compounds

381 against a reduction in specific SLs, such as 8-deoxylactucin-15-sulphate. Our work therefore

382 enables a refinement of breeding for metabolic composition in lettuce and directly relates

383 biochemical composition to consumer preference. Reducing the content of all sesquiterpene lactones would potentially decrease ability of the plant to defend itself from 
385 attack, thereby decreasing yield and shelf life, but our approach enables a balanced

386 breeding strategy by maximising the most sweet sugars and minimising only the most bitter

387 of the sesquiterpene lactones.

388

389 Acknowledgements

390 MJC would like to thank both Tozer Seeds Ltd and the BBSRC for their financial backing and

391 support of this work via a CASE studentship BB/G017670/1

392 
394 Baldwin, E. A., Goodner, K., \& Plotto, A. (2008). Interaction of volatiles, sugars, and acids on perception of tomato aroma and flavor descriptors. Journal of Food Science, 73, S294-307. Bartoshuk, L. M. (1975). Taste mixtures: Is mixture suppression related to compression? Physiology and Behavior, 14, 643-649.

Bartoshuk, L. M., Duffy, V. B., \& Miller, I. J. (1994). PTC/PROP tasting: Anatomy, psychophysics, and sex effects. Physiology and Behavior, 56, 1165-1171.

Behrens, M., Brockhoff, A., Batram, C., Kuhn, C., Appendino, G., \& Meyerhof, W. (2009). The human bitter taste receptor hTAS2R50 is activated by the two natural bitter terpenoids andrographolide and amarogentin. Journal of Agricultural and Food Chemistry, 57, 9860-9866.

Belitz, H. D., Grosch, W., \& Schieberle, P. (2009). Food Chemistry $4^{\text {th }}$ Ed, Springer-Verlag, Berlin, p. 1. Bennett, M. H., 1994. The phytoalexin response of lettuce to challenge by Botrytis cinerea, Bremia lactucae, and Pseudomonas syringae pv. phaseolicola. Physiological and Molecular Plant Pathology, 44, 321-333.

Bork, P. M., Schmitz, M. L., Kuhnt, M., Escher, C., \& Heinrich, M. (1997). Sesquiterpene lactone containing Mexican Indian medicinal plants and pure sesquiterpene lactones as potent inhibitors of transcription factor NF-[kappa]B. FEBS Letters, 402, 85-90.

Brockhoff, A., Behrens, M., Massarotti, A., Appendino, G., \& Meyerhof, W. (2007). Broad tuning of the human bitter taste receptor hTAS2R46 to various sesquiterpene lactones, clerodane and labdane diterpenoids, strychnine, and denatonium. Journal of Agricultural and Food Chemistry, 55, 62366243.

Brockhoff, A., Behrens, M., Niv, M. Y., \& Meyerhof, W. (2010). Structural requirements of bitter taste receptor activation. Proceedings of the National Academy of Sciences, 107, 11110-11115.

Casagrande, S. S., Wang, Y., Anderson, C., Gary, T. L. (2007). Have Americans Increased their fruit and vegetable intake?: The Trends Between 1988 and 2002. American Journal of Preventive Medicine, 32, 257-263.

Chadwick, M., Trewin, H., Gawthrop, F., \& Wagstaff, C. (2013). Sesquiterpenoid lactones: benefits to plants and people. International Journal of Molecular Science, 14, 12780-12805.

Cowan, M. M. (1999). Plant products as antimicrobial agents. Clinical Microbiology Reviews, 12, 564582.

Doty, R. L., Applebaum, S., Zusho, H., \& Settle, R. G. $(1985)$. Sex differences in odor identification ability: A cross-cultural analysis. Neuropsychologia, 23, 667-672.

Enneking, U., Neumann, C., \& Henneberg, S. (2007). How important intrinsic and extrinsic product attributes affect purchase decision. Food Quality and Preference, 18, 133-138.

García-Macías, P., Ordidge, M., Vysini, E., Waroonphan, S., Battey, N. H., Gordon, M. H., Hadley, P., John, P., Lovegrove, J. A., \& Wagstaffe, A. (2007). Changes in the flavonoid and phenolic acid contents and antioxidant activity of red leaf lettuce (Lollo Rosso) due to cultivation under plastic films varying in ultraviolet transparency. Journal of Agricultural and Food Chemistry, 55, 1016810172.

Giordano, O. S., Guerreiro, E., Pestchanker, M. J., Guzman, J., Pastor, D., \& Guardia, T. (1990). The gastric cytoprotective effect of several sesquiterpene lactones. Journal of Natural Products, 53, 803809.

Green, B. G., Lim, J., Osterhoff, F., Blacher, K., \& Nachtigal, D. (2010). Taste mixture interactions: suppression, additivity, and the predominance of sweetness. Physiology and Behavior, 101, 731-737. Green, B. G., Shaffer, G. S., \& Gilmore, M. M. (1993). Derivation and evaluation of a semantic scale of oral sensation magnitude with apparent ratio properties. Chemical Senses, 18, 683-702.

Guzman, M. L., Rossi, R. M., Karnischky, L., Li, X., Peterson, D. R., Howard, D. S., \& Jordan, C. T. (2005). The sesquiterpene lactone parthenolide induces apoptosis of human acute myelogenous leukemia stem and progenitor cells. Blood, 105, 4163-4169. 
Ivie, G. W., Witzel, D. A., \& Rushing, D. D. (1975). Toxicity and milk bittering properties of tenulin, the

443 major sesquiterpene lactone constituent of Helenium amarum (bitter sneezeweed). Journal of

444 Agricultural and Food Chemistry, 23, 845-849.

445 Keast, R. S., \& Breslin, P. A. (2003). An overview of binary taste-taste interactions. Food Quality and

446 Preference, 14, 111-124.

447 Kim, U., Wooding, S., Ricci, D., Jorde, L. B., \& Drayna, D. (2005). Worldwide haplotype diversity and

448 coding sequence variation at human bitter taste receptor loci. Human Mutation, 26, 199-204. Koehler, P. E. \& Kays S. J. (1991). Sweet potato flavor: Quantitative and qualitative assessment of optimum sweetness. Journal of Food Quality, 14, 241-249.

Koul, O. (2008). Phytochemicals and insect control: an antifeedant approach. Critical Reviews in Plant Sciences, 27, 1-24.

Meyerhof, W., Batram, C., Kuhn, C., Brockhoff, A., Chudoba, E., Bufe, B., Appendino, G., \& Behrens, M. (2010). The Molecular Receptive Ranges of Human TAS2R Bitter Taste Receptors. Chemical Senses, 35, 157-170.

Meyers, B., \& Brewer, M. S. (2008). Sweet taste in man: A review. Journal of Food Science, 73, R81R90.

McMath, K., Paterson, V., Young, H., \& Ball, R. (1991). Factors affecting the sensory perception of sweetness and acidity in kiwifruit. II International Symposium on Kiwifruit 297, pp. 489-500. Mennella, J. A., Pepino, M. Y., Duke, F. F., \& Reed, D. R. (2010). Age modifies the genotypephenotype relationship for the bitter receptor TAS2R38. BMC Genetics, 11, 60.

Oh, M. M., Trick, H. N., \& Rajashekar, C. B. (2009). Secondary metabolism and antioxidants are involved in environmental adaptation and stress tolerance in lettuce. Journal of Plant Physiology, 166, 180-191.

Pangborn, R. (1963). Relative taste intensities of selected sugars and organic acids. Journal of Food Science, 28, 726-733.

Peters, A. M. \& van Amerongen, A. (1998). Relationship between levels of sesquiterpene lactones in chicory and sensory evaluation. Journal of the American Society for Horticultural Science, 123, 326329.

470 Price, K., DuPont, M. S., Shepherd, R., Chan, H.W.-S., \& Fenwick, G. (1990). Relationship between the 471 chemical and sensory properties of exotic salad crops - coloured lettuce (Lactuca sativa) and chicory (Chicorium intybus). Journal of the Science of Food and Agriculture, 53, 185-192. Rogers, S. \& Pryer, J. A. (2012). Who consumed 5 or more portions of fruit and vegetables per day in 1986-1987 and in 2000-2001? Public Health Nutrition, 15, 1240-1247.

Sayyah, M., Hadidi, N., \& Kamalinejad, M. (2004). Analgesic and anti-inflammatory activity of Lactuca sativa seed extract in rats. Journal of Ethnopharmacology, 92, 325-329.

Seo, M. W., Yang, D. S., Kays, S. J., Lee, G. P., \& Park, K. W. (2009). Sesquiterpene lactones and bitterness in Korean leaf lettuce cultivars. HortScience, 44, 246-249.

Sessa, R. A., Bennett, M. H., Lewis, M. J., Mansfield, J. W., \& Beale, M. H. (2000). Metabolite profiling of sesquiterpene lactones from Lactuca species. Journal of Biological Chemistry, 275, 26877-26884. Spencer, J. P. E., Vauzour, D., \& Rendeiro, C. (2009). Flavonoids and cognition: The molecular mechanisms underlying their behavioural effects. Archives of Biochemistry and Biophysics, 492, 1-9. Tieman, D., Bliss, P., McIntyre, L. M., Blandon-Ubeda, A., Bies, D., Odabasi, A. Z., Rodríguez, G. R., van der Knaap, E., Taylor, M. G., \& Goulet, C. (2012). The chemical interactions underlying tomato flavor preferences. Current Biology, 22, 1035-1039.

Van Beek, T. A., Maas, P., King, B. M., Leclercq, E., Voragen, A. G. J., \& De Groot, A. (1990). Bitter sesquiterpene lactones from chicory roots. Journal of Agricultural and Food Chemistry, 38, 10351038.

Wedge, D. E., Galindo, J. C. G., \& Macías, F. A. (2000). Fungicidal activity of natural and synthetic sesquiterpene lactone analogs. Phytochemistry, 53, 747-757. 
491 Wingler, A., Purdy, S., Maclean, J. A., \& Portau, N. (2006). The role of sugars in integrating environmental signals during the regulation of leaf senescence. Journal of Experimental Botany, 57, 493 391-399.

494 Wink, M. (1988). Plant breeding: importance of plant secondary metabolites for protection against

495 pathogens and herbivores. Theoretical and Applied Genetics, 75, 225-233.

496

497

498 
500 Table S1. Buffer conditions and gradient for HPLC used for SL Analysis

\begin{tabular}{rrr}
\hline Time (mins) & \% A & \% B \\
\hline 0 & 5 & 95 \\
5 & 5 & 95 \\
40 & 50 & 50 \\
55 & 100 & 0 \\
59.9 & 100 & 0 \\
60 & 5 & 95 \\
\hline A $=50 \%$ Acetonitrile $50 \% \mathrm{H} 20$ & $0.1 \% \mathrm{HCl}$ &
\end{tabular}

501

$B=95 \% \mathrm{H} 2 \mathrm{O}, 5 \%$ Methanol, $0.1 \% \mathrm{HCl}$

502 Table S2. Concentration of sugars and SLs present in selected lines. Values given in

$503 \mu \mathrm{g} / \mathrm{g}$ dry weight. SLs were analysed by HPLC and confirmed by MS. RILs were selected for

504 extremes in concentrations of the compounds listed from within the whole population of 96

505 lines. Table A gives the mean values, Table B gives the raw values, mean values, and

506 standard error of mean ( $n=4$ biologically distinct samples). Quantity of sesquiterpene

507 lactones in lettuce RILs was relative to the wild parent L. serriola UC96US23, which was

508 given a value of 100 in each case. Values were determined from total peak area. Sugars

509 were analysed by high throughput plate assay as described in the methods.

510 Table A.

\begin{tabular}{ccccccccc}
\hline RIL & $\mathbf{1 9}$ & $\mathbf{4 1}$ & $\mathbf{6 1}$ & $\mathbf{8 9}$ & $\mathbf{9 4}$ & $\mathbf{1 2 1}$ & $\mathbf{1 2 2}$ & $\mathbf{1 2 3}$ \\
\hline Total Sugar & 1433.0 & 635.6 & 583.0 & 1178.0 & 1363.0 & 1036.0 & $\mathbf{7 6 6 . 1}$ & 568.0 \\
Total SL & 70.8 & 262.1 & 32.3 & 93.3 & 529.5 & 56.1 & 222.5 & 803.8 \\
Fructose & 279.7 & 189.2 & 137.0 & 443.3 & 475.1 & 388.0 & 206.6 & 214.8 \\
Sucrose & 436.3 & 252.4 & 160.0 & 264.0 & 313.7 & 222.4 & 301.9 & 127.2 \\
Glucose & 605.3 & 149.2 & 256.0 & 397.3 & 481.1 & 377.1 & 184.9 & 182.4 \\
Lactucin & 7.6 & 14.8 & 1.4 & 5.1 & 7.3 & 9.0 & 16.2 & 11.8 \\
Lactucopicrin & 18.2 & 27.7 & 4.9 & 6.9 & 14.5 & 19.9 & 35.9 & 20.2 \\
8-deoxylactucin-15-sulphate & 0.6 & 23.9 & 4.0 & 14.4 & 432.6 & 17.8 & 8.3 & 682.5 \\
Lactucin-15-oxalate & 8.8 & 71.2 & 3.8 & 21.9 & 28.6 & 1.9 & 78.0 & 43.6 \\
Lactucopicrin-15-oxalate & 28.7 & 85.7 & 18.1 & 34.2 & 46.3 & 7.3 & 83.9 & 45.4 \\
15-p- & & & & & & & & \\
hydroxylphenylacetyllactucin- & 6.9 & 38.7 & 0.1 & 10.9 & 0.2 & 0.1 & 0.3 & 0.4 \\
8-sulphate & & & & & & & &
\end{tabular}

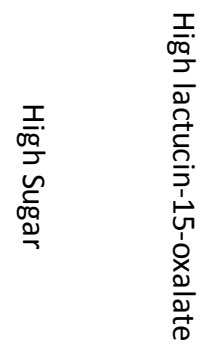

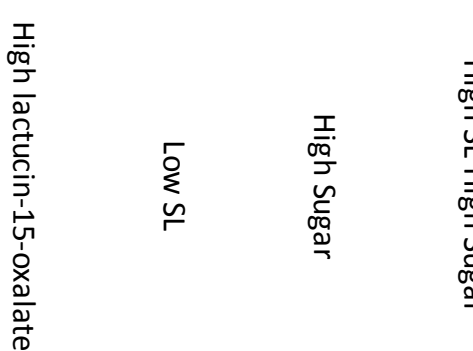

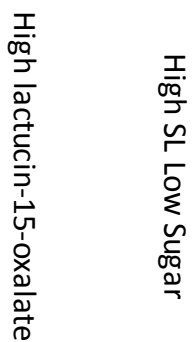


512 Table B.

Raw HPLC-MS relative quantities of sesquiterpenes

513 p-15-

Line Lactucin Lactucopicrin hydroxyphenyllacetyllactu $\begin{array}{ccc}\text { 8-deoxylactucin- } & \text { lactucopicrin-15- lactucin-15- } \\ \text { 15-oxalate } & \text { oxalate } & \text { oxalate }\end{array}$ Total SL cin-8-sulphate

\begin{tabular}{|c|c|c|c|c|c|c|c|}
\hline 19 & 1.92 & 6.01 & 3.59 & 0.42 & 20.01 & 6.03 & 37.98 \\
\hline 19 & 4.97 & 12.95 & 2.08 & 0.24 & 3.10 & 1.42 & 24.76 \\
\hline 19 & 7.34 & 15.21 & 3.30 & 0.18 & 6.70 & 2.67 & 35.40 \\
\hline 19 & 0.92 & 2.16 & 4.83 & 0.45 & 27.62 & 7.54 & 43.52 \\
\hline 41 & 17.96 & 32.50 & 41.13 & 28.72 & 87.62 & 80.80 & 288.74 \\
\hline 41 & 2.52 & 3.96 & 11.13 & 4.99 & 26.27 & 19.41 & 68.28 \\
\hline 41 & 1.79 & 5.09 & 5.76 & 2.12 & 14.71 & 6.65 & 36.12 \\
\hline 61 & 0.68 & 2.83 & 0.03 & 1.47 & 9.97 & 1.73 & 16.70 \\
\hline 61 & 1.62 & 4.34 & 0.06 & 1.48 & 11.58 & 3.48 & 22.57 \\
\hline 61 & 0.34 & 1.88 & 0.05 & 4.09 & 8.76 & 1.66 & 16.79 \\
\hline 61 & 0.17 & 0.85 & 0.07 & 0.91 & 5.87 & 0.75 & 8.63 \\
\hline 89 & 3.69 & 6.30 & 5.20 & 3.92 & 9.71 & 5.40 & 34.22 \\
\hline 89 & 2.88 & 2.75 & 4.85 & 7.45 & 17.18 & 12.79 & 47.90 \\
\hline 89 & 2.87 & 3.08 & 7.07 & 13.52 & 25.61 & 18.23 & 70.38 \\
\hline 89 & 0.73 & 1.67 & 4.60 & 3.95 & 15.88 & 7.30 & 34.13 \\
\hline 94 & 1.75 & 6.74 & 0.08 & 579.99 & 11.34 & 22.27 & 622.18 \\
\hline 94 & 8.53 & 13.40 & 0.20 & 84.84 & 37.31 & 19.27 & 163.55 \\
\hline 94 & 2.70 & 6.21 & 0.01 & 82.42 & 17.80 & 6.71 & 115.85 \\
\hline 94 & 1.56 & 2.70 & 0.14 & 117.92 & 26.10 & 9.02 & 157.44 \\
\hline 121 & 1.42 & 5.50 & 0.03 & 8.90 & 3.43 & 0.76 & 20.04 \\
\hline 121 & 11.40 & 24.03 & 0.13 & 0.87 & 1.52 & 0.33 & 38.28 \\
\hline 121 & 0.97 & 2.51 & 0.02 & 20.18 & 7.98 & 2.02 & 33.68 \\
\hline 121 & 4.31 & 7.75 & 0.05 & 5.73 & 1.72 & 0.66 & 20.22 \\
\hline 122 & 4.04 & 18.54 & 0.08 & 8.83 & 25.63 & 59.51 & 116.62 \\
\hline 122 & 6.35 & 11.40 & 0.10 & 1.01 & 17.91 & 8.83 & 45.59 \\
\hline 122 & 18.76 & 35.94 & 0.35 & 5.27 & 101.36 & 76.29 & 237.96 \\
\hline 122 & 3.22 & 5.86 & 0.08 & 1.48 & 22.90 & 11.29 & 44.83 \\
\hline 123 & 2.66 & 8.81 & 0.26 & 624.57 & 15.17 & 32.01 & 683.48 \\
\hline 123 & 11.39 & 16.20 & 0.11 & 71.41 & 18.08 & 12.58 & 129.78 \\
\hline 123 & 5.50 & 8.44 & 0.31 & 249.96 & 33.31 & 26.53 & 324.05 \\
\hline 123 & 3.99 & 6.96 & 0.18 & 149.16 & 24.16 & 16.09 & 200.52 \\
\hline \multicolumn{8}{|c|}{ Average HPLC-MS relative quantities of sesquiterpenes } \\
\hline 19 & 3.79 & 9.08 & 3.45 & 0.32 & 14.36 & 4.41 & 4.41 \\
\hline 41 & 7.42 & 13.85 & 19.34 & 11.94 & 42.87 & 35.62 & 35.62 \\
\hline 61 & 0.70 & 2.47 & 0.05 & 1.99 & 9.05 & 1.91 & 1.91 \\
\hline 89 & 2.54 & 3.45 & 5.43 & 7.21 & 17.09 & 10.93 & 10.93 \\
\hline 94 & 3.63 & 7.26 & 0.11 & 216.29 & 23.14 & 14.32 & 14.32 \\
\hline 121 & 4.52 & 9.95 & 0.06 & 8.92 & 3.66 & 0.94 & 0.94 \\
\hline 122 & 8.09 & 17.93 & 0.15 & 4.15 & 41.95 & 38.98 & 38.98 \\
\hline 123 & 5.88 & 10.10 & 0.22 & 273.78 & 22.68 & 21.80 & 21.80 \\
\hline
\end{tabular}

SEM of HPLC-MS relative quantities of sesquiterpenes

\begin{tabular}{cccccccc}
\hline $\mathbf{1 9}$ & 1.46 & 3.03 & 0.56 & 0.07 & 5.72 & 1.43 & 3.94 \\
\hline $\mathbf{4 1}$ & 5.27 & 9.33 & 11.01 & 8.43 & 22.63 & 22.89 & 79.39 \\
\hline $\mathbf{6 1}$ & 0.32 & 0.74 & 0.01 & 0.71 & 1.20 & 0.57 & 2.86 \\
\hline $\mathbf{8 9}$ & 0.63 & 1.00 & 0.56 & 2.26 & 3.27 & 2.89 & 8.54 \\
\hline $\mathbf{9 4}$ & 1.65 & 2.23 & 0.04 & 121.50 & 5.61 & 3.80 & 119.61 \\
\hline $\mathbf{1 2 1}$ & 2.41 & 4.82 & 0.02 & 4.10 & 1.50 & 0.37 & 4.67 \\
\hline $\mathbf{1 2 2}$ & 3.62 & 6.54 & 0.07 & 1.83 & 19.87 & 17.05 & 45.47 \\
\hline $\mathbf{1 2 3}$ & 1.93 & 2.07 & 0.05 & 122.51 & 4.01 & 4.51 & 123.07 \\
\hline
\end{tabular}

Sugar content (mg/g DW)

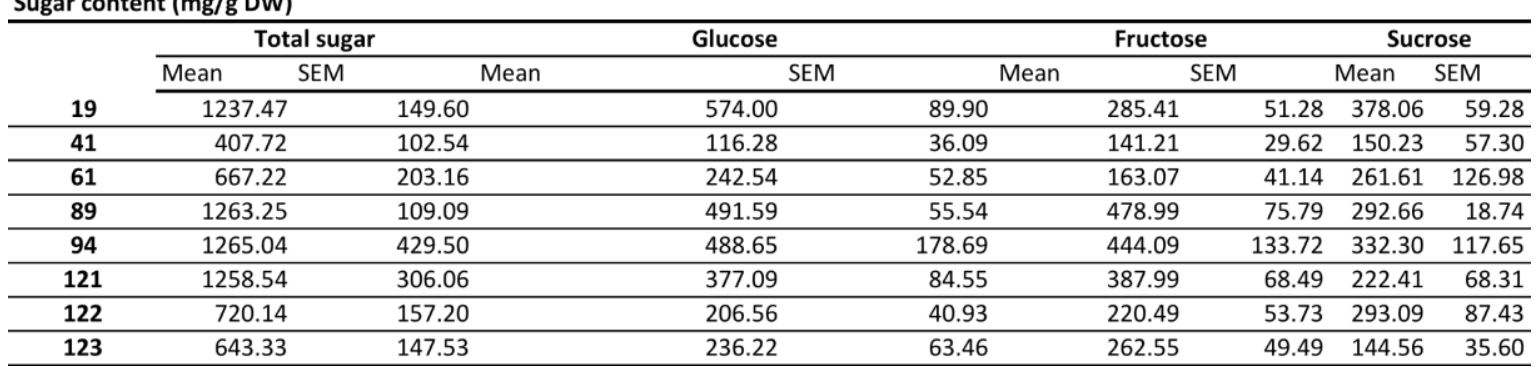


Figure 1. Mean scores for bitterness, sweetness, aftertaste, and liking. Consumer scores

516 for sweetness and bitterness (A) and for aftertaste perception and consumer liking by RIL.

517 (B) Bitterness and sweetness scores as log mean values from the LMS scale as described in

518 Green (1993). Aftertaste was assessed on a 5 point hedonic scale, while liking was

519 measured on a 9 point hedonic scale. Error bars show standard error, $n=43$. Categories a-d denote significantly different groupings as determined by Kruskal-Wallis, Dunn's procedure.

\section{Figure 2. Quantified Sugar Concentration vs Perceived Sweetness in Lettuce Lines}

522 Total sugar $(A)(r=0.1747 \mathrm{P}<0.0001)$ correlates to perceived sweetness less well than does glucose $(D)(r=0.2266 P<0.0001)$. Fructose $(B)$ is not significant, owing to RIL 61 , which has

524 the lowest levels of fructose, yet the highest perceived sweetness. Sucrose $(C)(r=0.1543 p-$ 0.0041 ) has less correlation to perceived sweetness that does glucose despite a higher relative sweetness. Taking into account relative sweetness (E) highlights the lack of sweetness suppression in RILs 61 and 122.

Figure 3. Quantified sesquiterpene concentration vs perceived bitterness in lettuce lines.

Total SL $(A)(r=0.56 P<0.0001)$ correlates best. The most significant individual $S L$ is 8-

530 deoxylactucin-15-sulphate $(B)(r=0.3403 \mathrm{P}<0.0001)$ possibly due to the very high levels observed in some samples. Lactucin and lactucopicrin ( $C$ and $D)$ had equal effect as scored by consumers $(r=0.1817 \mathrm{P}=0.0007)$ and were each less bitter than their oxalates $(\mathrm{E}$ and $\mathrm{F})$. Lactucin-15-oxalate $(r=0.1986 \mathrm{P}=0.0002)$ was less bitter than lactucopicrin-15-

534 oxalate $(r=0.226 \mathrm{P}<0.0001)$ as was expected. 15 - $p$-hydroxylphenylacetyllactucin-8-sulphate was not significantly correlated to bitterness in our samples. 
537 Sweetness (A) positively correlates with liking, ( $r=0.4026 \mathrm{P}<0.0001)$, while bitterness $(\mathrm{B})$ and

538 aftertaste (C) negatively correlate ( $r=-0.56 \mathrm{P}<0.0001$ and $\mathrm{r}=0.3075 \mathrm{P}<0.0001$ respectively).

539 As all results are so significant, it is clear to us that consumers have a strong and reliable

540 aversion to bitterness and preference for sweetness in lettuce.

\section{Figure 5. Consumer Preference Map}

$54228.74 \%$ of variance in reported liking is a consequence of sweet-bitter balance. The

543 secondary and subsequent components relate to traits which were not assessed in this

544 study, but participants were able to distinguish lines based upon this, with RILs 122 and 94

545 driving this trait positively and negatively respectively.

\section{$546 \quad$ Figure 6. Consumer Perception vs Predicted Perception}

547 Taking into account the ratio of sugars to sesquiterpene lactones take into account the

548 mixing suppression to an extent and corrects outliers affected by this. Using this method

549 the correlation to preference (A) was no longer significant, and the correlation to sweetness

550 dropped $(B),(r=0.171 P=0.0015)$ though the correlation to bitterness $(C)$ remained strong

$551 \quad(r=-0.2803 P<0.0001)$.

552 Supplementary Figure 1. MS/MS fragmentation of assessed sesquiterpene lactones.

$553 \mathrm{MS} / \mathrm{MS}$ fragmentation spectra of each sesquiterpene lactone, determined by Agilent 1100

554 HPLC with QToFMS.

555

556 

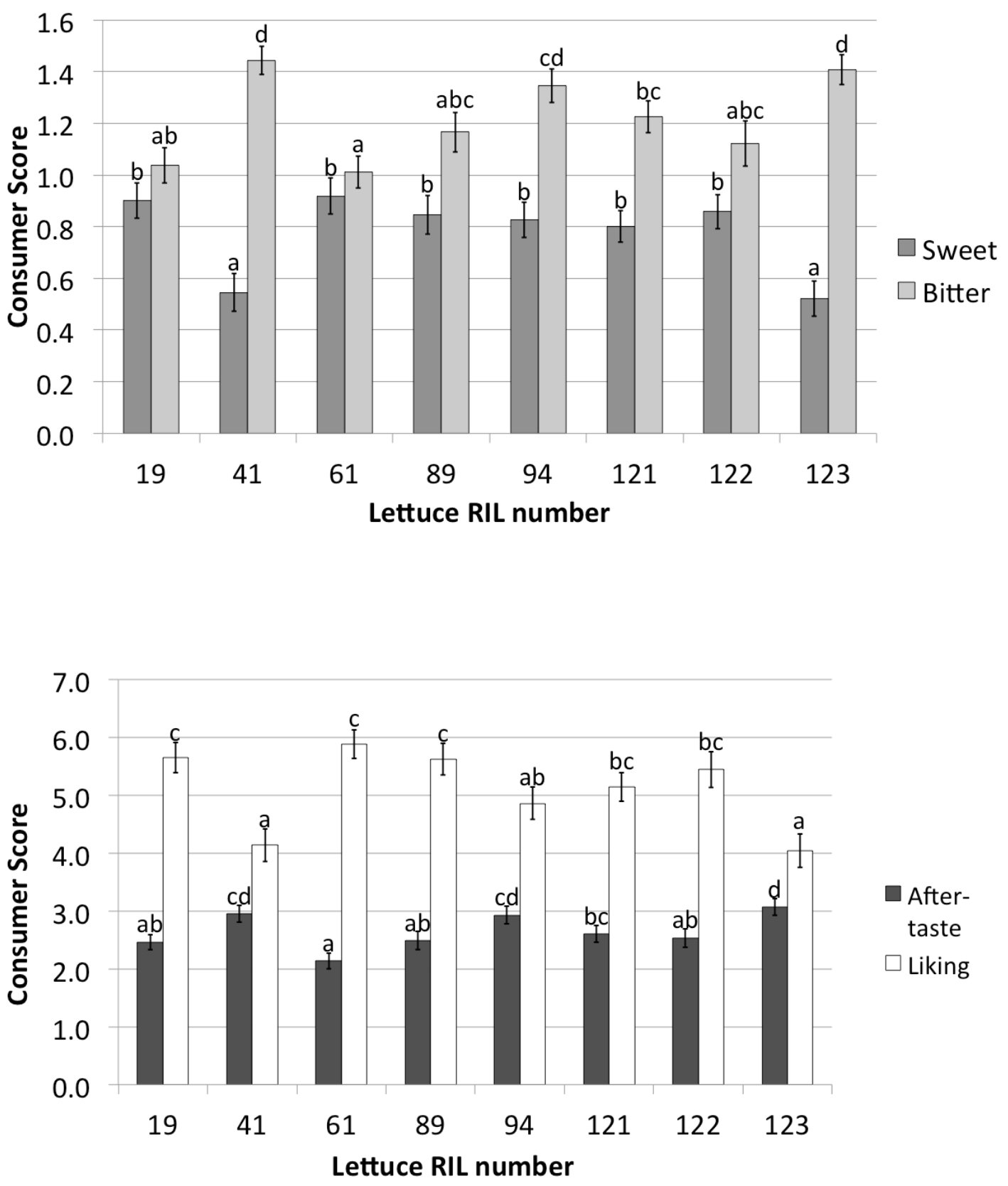

Figure 1. Mean scores for bitterness, sweetness, aftertaste, and liking. Consumer scores for sweetness and bitterness (A) and for aftertaste perception and consumer liking by RIL. (B) Bitterness and sweetness scores as log mean values from the LMS scale as described in Green (1993). Aftertaste was assessed on a 5 point hedonic scale, while liking was measured on a 9 point hedonic scale. Error bars show standard error, $n=43$. Categories a-d denote significantly different groupings as determined by Kruskal-Wallis, Dunn's procedure. 

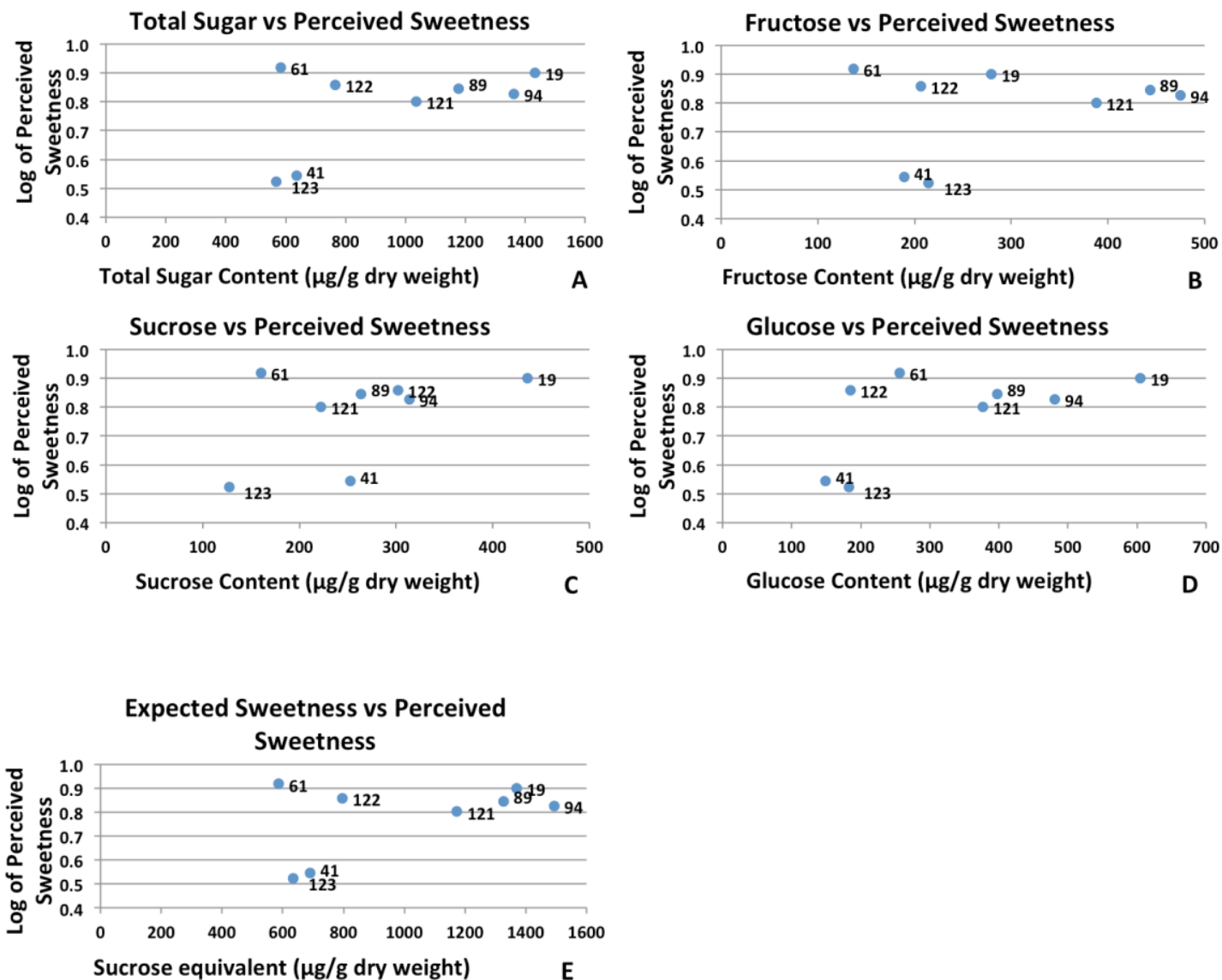

573 Figure 2. Quantified Sugar Concentration vs Perceived Sweetness in Lettuce Lines

574 Total sugar $(\mathrm{A})(\mathrm{r}=0.1747 \mathrm{P}<0.0001)$ correlates to perceived sweetness less well than 575 does glucose (D) $(r=0.2266 \mathrm{P}<0.0001)$. Fructose (B) is not significant, owing to RIL 61, 576 which has the lowest levels of fructose, yet the highest perceived sweetness. Sucrose 577 (C) $(r=0.1543 \mathrm{p}=0.0041)$ has less correlation to perceived sweetness that does glucose 578 despite a higher relative sweetness. Taking into account relative sweetness (E) highlights the lack of sweetness suppression in RILs 61 and 122. 
Total SL vs Perceived Bitterness
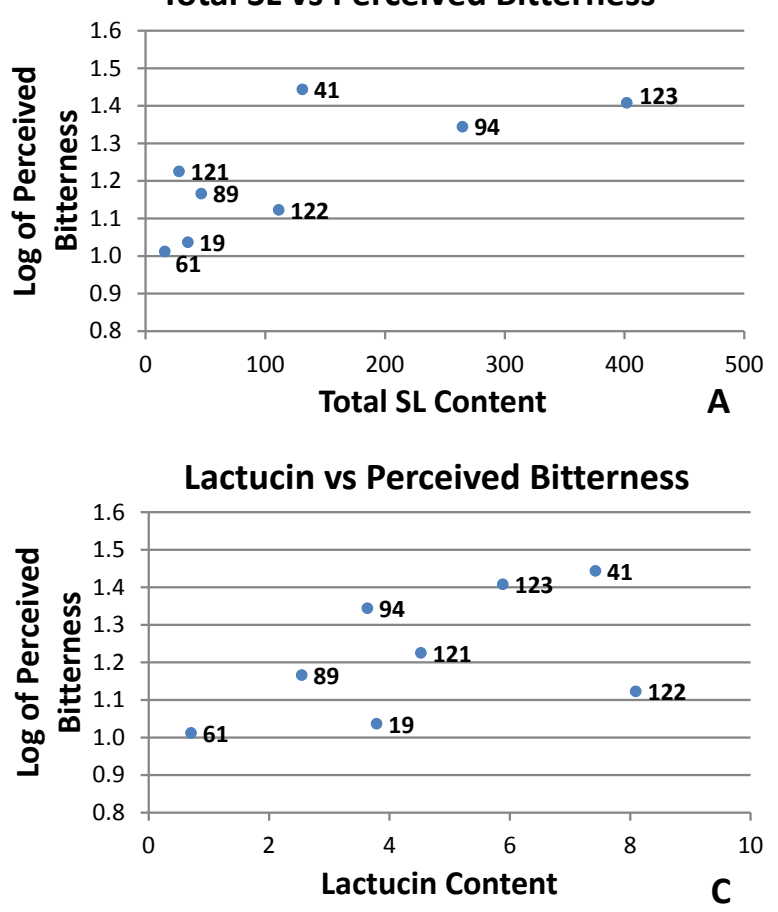

Lactucin-15-oxalate vs Perceived Bitterness

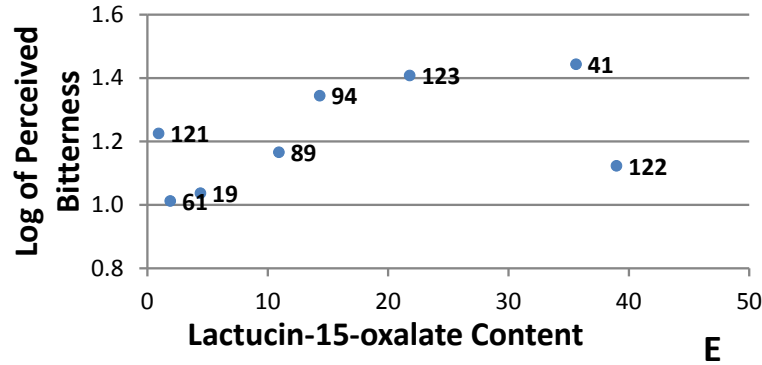

15-p-hydroxylphenylacetyllactucin-8sulphate vs Perceived Bitterness

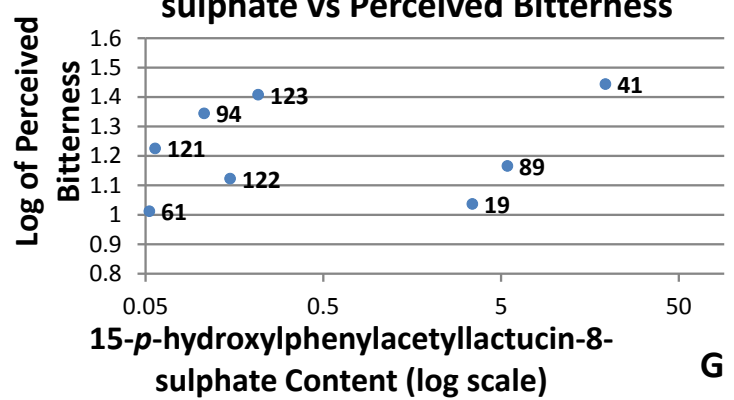
in our samples. 8-deoxylactucin-15-oxalate vs Perceived Bitterness
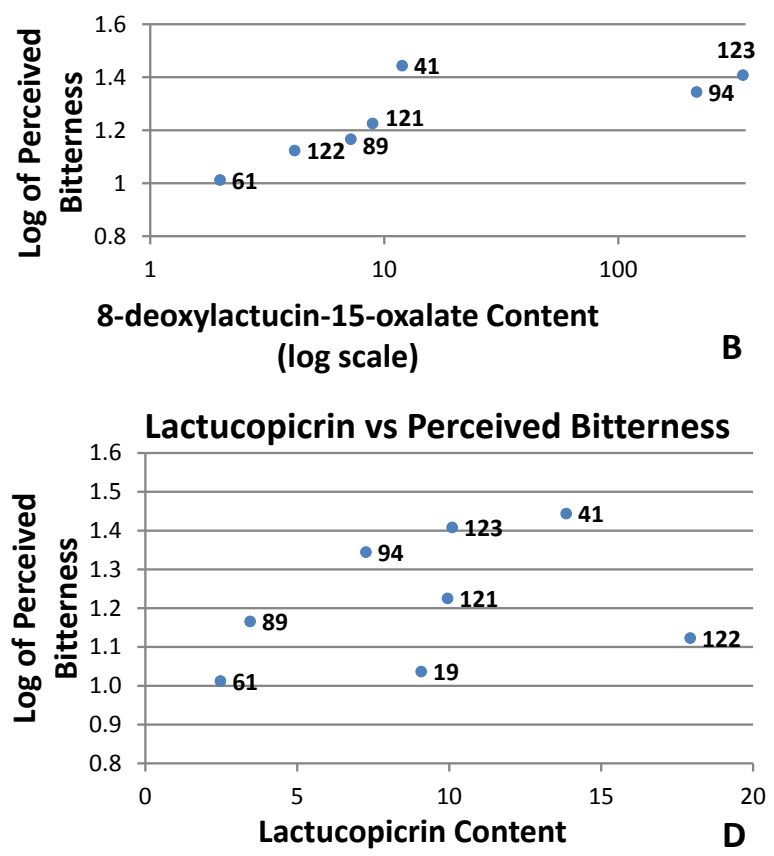

Lactucopicrin-15-oxalate vs Perceived Bitterness

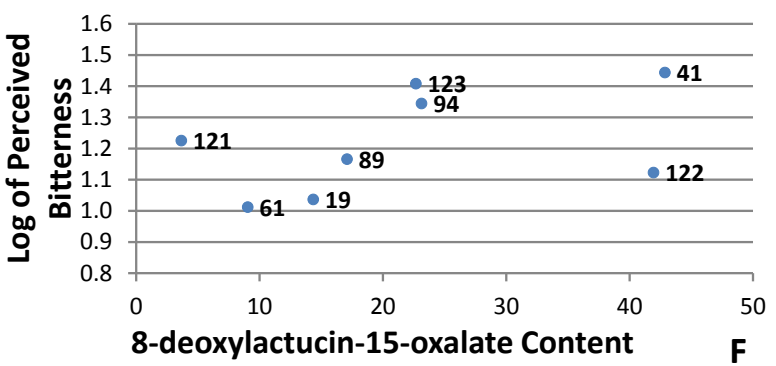

Figure 3. Quantified sesquiterpene concentration vs perceived bitterness in lettuce lines. Total SL $(\mathrm{A})(\mathrm{r}=0.56 \mathrm{P}<0.0001)$ correlates best. The most significant individual SL is 8-deoxylactucin-15-sulphate $(\mathrm{B})(\mathrm{r}=0.3403 \mathrm{P}<0.0001)$ possibly due to the very high levels observed in some samples. Lactucin and lactucopicrin ( $C$ and $D$ ) had equal effect as scored by consumers $(\mathrm{r}=0.1817 \mathrm{P}=0.0007)$ and were each less bitter than their oxalates ( $\mathrm{E}$ and $\mathrm{F})$. Lactucin-15-oxalate $(\mathrm{r}=0.1986 \mathrm{P}=0.0002)$ was less bitter than lactucopicrin-15-oxalate( $\mathrm{r}=0.226 \quad \mathrm{P}<0.0001) \quad$ as was expected. 15-phydroxylphenylacetyllactucin-8-sulphate was not significantly correlated to bitterness 

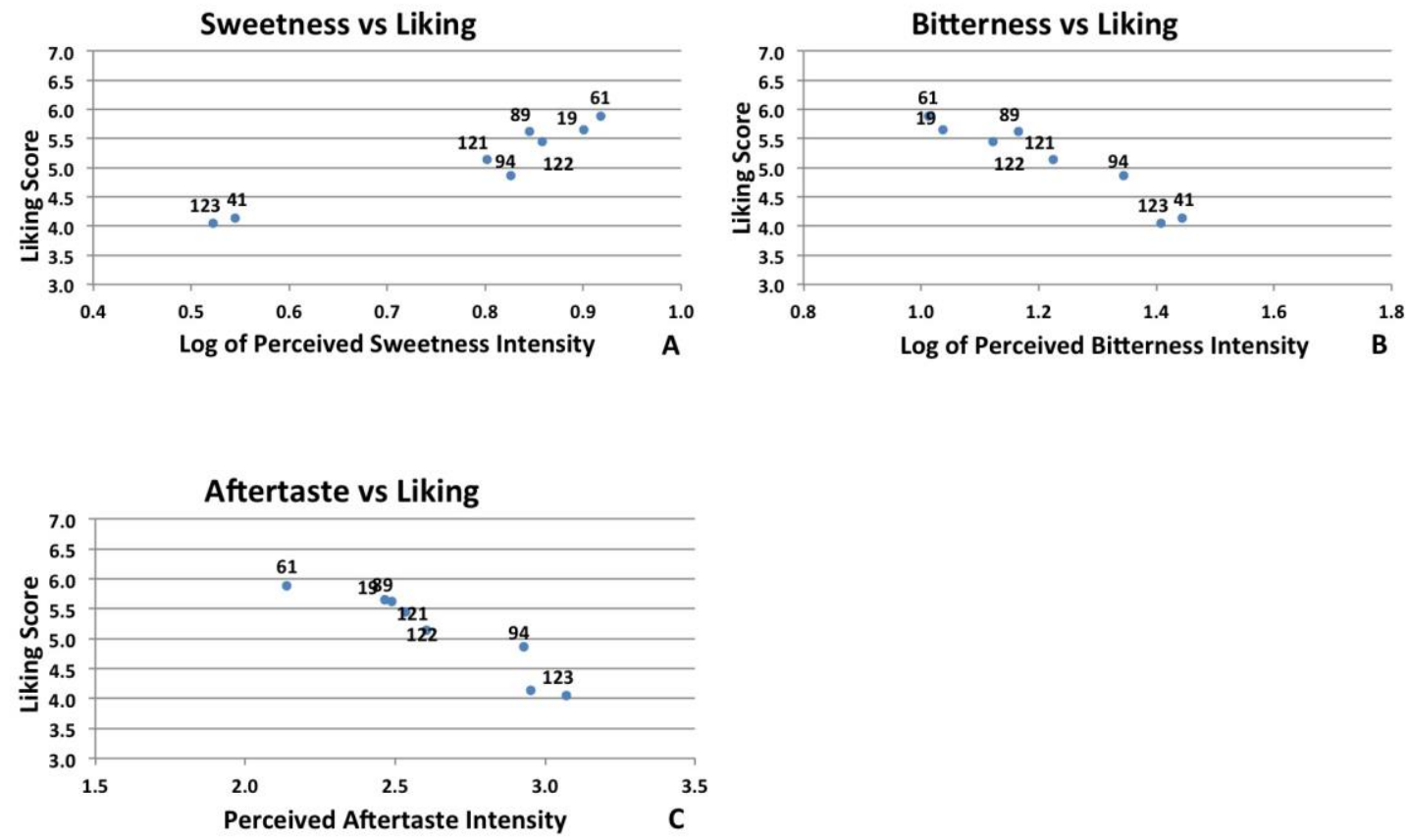

593

594 Figure 4. Perceived taste parameters vs liking for lettuce lines

595 Sweetness (A) positively correlates with liking, $(\mathrm{r}=0.4026 \mathrm{P}<0.0001)$, while bitterness

596 (B) and aftertaste (C) negatively correlate $(r=-0.56 \mathrm{P}<0.0001$ and $\mathrm{r}=0.3075 \mathrm{P}<0.0001$

597 respectively). As all results are so significant, it is clear to us that consumers have a 598 strong and reliable aversion to bitterness and preference for sweetness in lettuce.

599

600

601

602 


\section{Preference Plot (axes F1 and F2)}

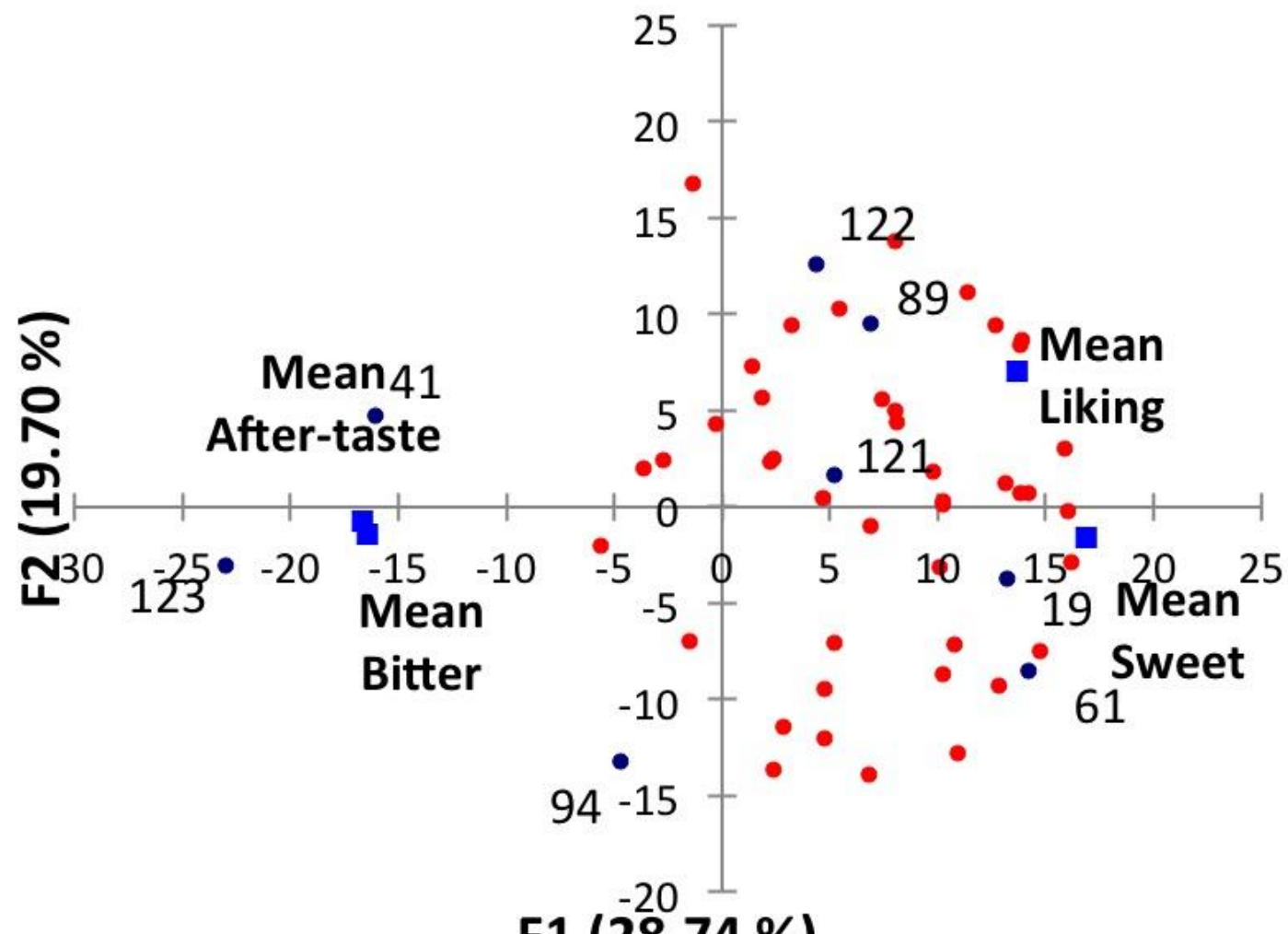

603

F1 (28.74\%)

604 Figure 5. Consumer Preference Map

$60528.74 \%$ of variance in reported liking is a consequence of sweet-bitter balance. The 606 secondary and subsequent components relate to traits which were not assessed in this 607 study, but participants were able to distinguish lines based upon this, with RILs 122 and 94 608 driving this trait positively and negatively respectively.

609 

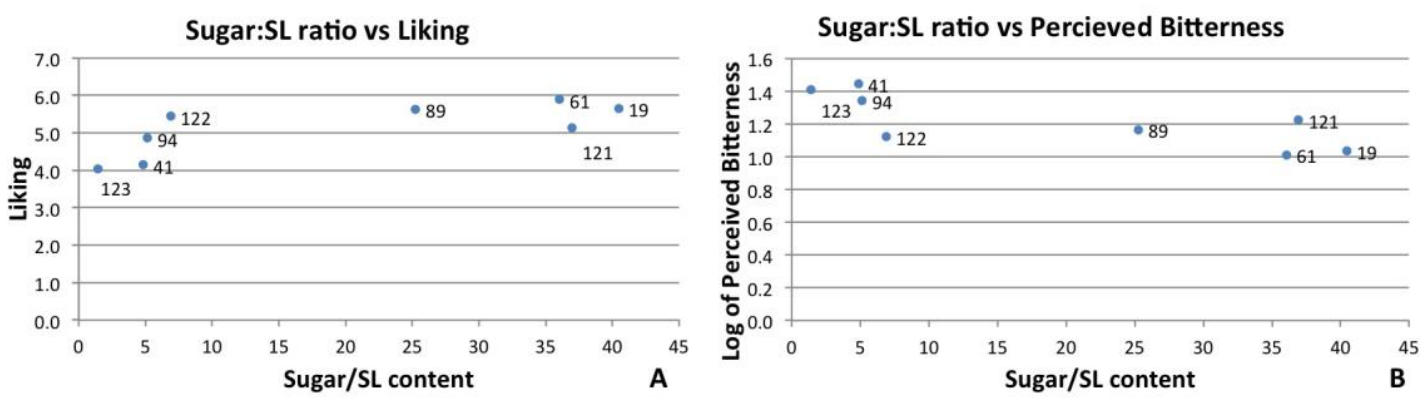

612

613

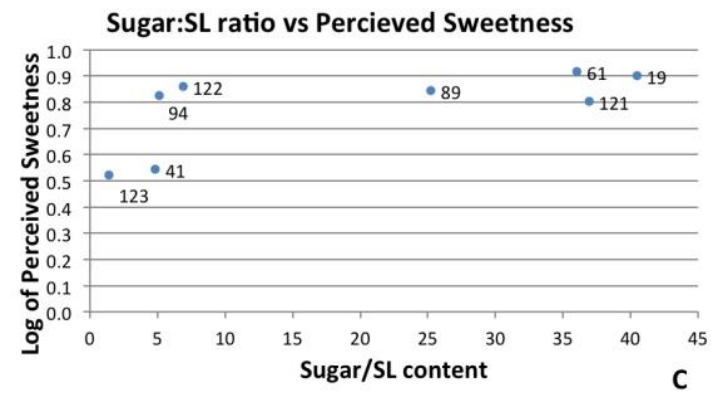

$614 \quad$ Figure 6. Consumer Perception vs Predicted Perception

615 Taking into account the ratio of sugars to sesquiterpene lactones take into account the 616 mixing suppression to an extent and corrects outliers affected by this. Using this method 617 the correlation to preference $(A)$ was no longer significant, and the correlation to sweetness 618 dropped $(B),(r=0.171 P=0.0015)$ though the correlation to bitterness $(C)$ remained strong $619 \quad(r=-0.2803 P<0.0001)$.

620 


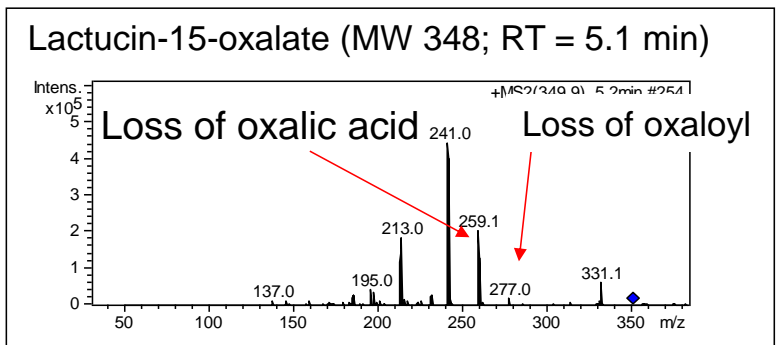

8-Deoxylactucin-15-oxalate (MW 332; RT = $13.3 \mathrm{~min}$ )

Intens. -7 +MS2(332.5), 13.4m $\#$ m $\# 47$

Loss of oxalic acid 243.1 Loss of oxaloyl

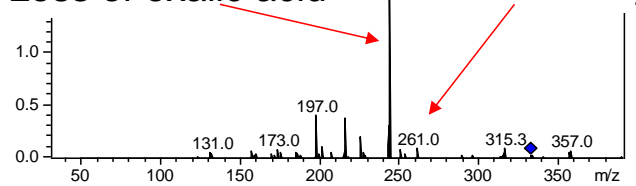

Lactucopicrin-15-oxalate

(MW 482; RT = $15.8 \mathrm{~min}$ )

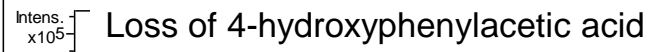

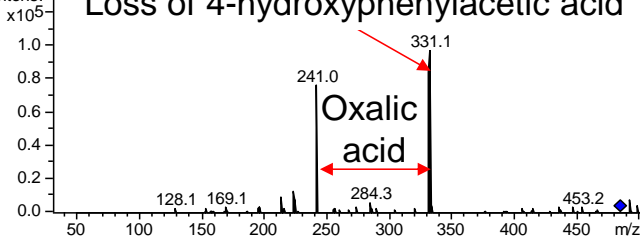

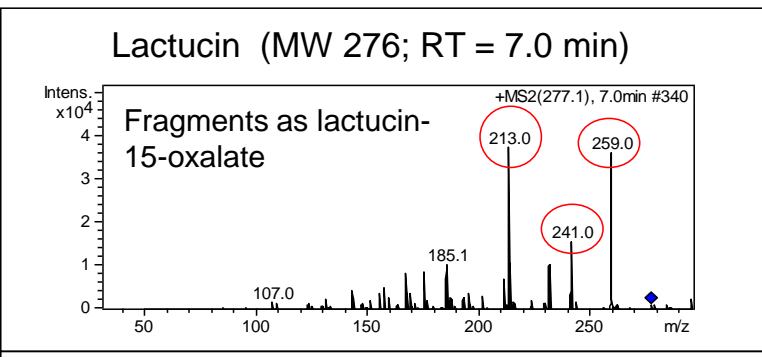

15-p-Hydroxyphenylacetyllactucin-8-sulfate (MW 490; RT = $15.5 \mathrm{~min}$ )

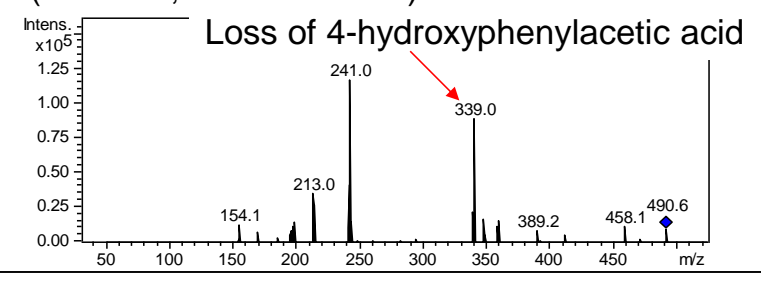

Lactucopicrin (MW 410; RT = $17.3 \mathrm{~min}$ )

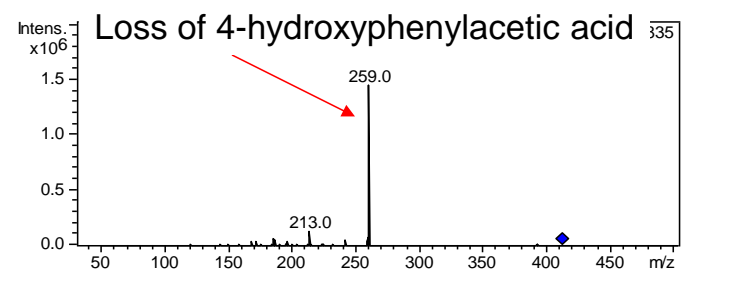

624

625 Supplementary Figure 1. MS/MS fragmentation of assessed sesquiterpene lactones.

626 MS/MS fragmentation spectra of each sesquiterpene lactone, determined by Agilent 1100

627 HPLC with QToFMS.

628

629 